\title{
Reduktion der Ansprechzeit Hydrogel-basierter Sensoren
}

\author{
Simon Binder ${ }^{1}$, Andreas T. Krause ${ }^{2}$, Gerald Gerlach ${ }^{1}$ \\ ${ }^{1}$ Institut für Festkörperelektronik, Technische Universität Dresden, 01062 Dresden, Deutschland \\ ${ }^{2}$ Leibniz-Institut für Polymerforschung e.V., 01005 Dresden, Deutschland \\ simon.binder@tu-dresden.de
}

\section{Zusammenfassung}

Die Leistungsfähigkeit Hydrogel-basierter Sensoren im Vergleich zu herkömmlichen biochemischen Sensoren ist bislang vor allem durch deren lange Ansprechzeiten eingeschränkt. Ursache der hohen Zeitkonstanten sind die für Polymere bzw. Hydrogele typischen Diffusionsprozesse, welche beim Quellen und Entquellen des Hydrogels als chemomechanischem Wandler zum Tragen kommen. In dieser Arbeit soll die Anwendung der Kraftkompensationsmethode dem entgegenwirken.

Dazu wird in Verbindung mit einem MEMS-Drucksensor als mechanoelektrischem Wandler eine neue Methode der Kraftkompensation vorgestellt. Grundlage dieses Ansatzes bildet ein bisensitives Hydrogel, welches die Aktorfunktion zur aktiven Kompensation durch einen Temperatureintrag bereits in sich trägt. Im Gegensatz zu anderen Kompensationsmethoden, die die Einkopplung einer externen Kraft über einen Aktor hoher Energiedichte erfordern, benötigt diese Messmethode lediglich eine Temperiereinheit in Form eines Peltierelements.

Durch die Anwendung der Kompensationsmethode wird das Hydrogelvolumen konstant gehalten. Damit werden langwierige Diffusionsprozesse unterdrückt und so schließlich die Ansprechzeit verkürzt. Durch die Verlagerung der Aktorfunktion in das sensitive Hydrogel hinein wird zusätzlich eine Vereinfachung des Aufbaus erzielt. Da das Materialvolumen stets nahezu konstant bleibt und damit nur geringe mechanische Deformationen auftreten, erhöht sich die Zuverlässigkeit und Langzeitstabilität dieses biochemischen Sensors.

Keywords: Kraftkompensation,biochemische Sensoren,Drucksensoren,Hydrogel-basierte Mikrosysteme,chemomechanische Wandler

\section{Einleitung}

Hydrogel-basierte Sensoren

Smarte Hydrogele zeichnen sich durch die Eigenschaft aus, abhängig von einem äußeren Stimulus, wie Temperatur, pH-Wert oder lonenkonzentration, in einer Messlösung spezifisch zu quellen oder entquellen. Jedem Stimuluswert ist ein reversibler Gleichgewichtsquellungszustand zuordenbar. Solche Stimulus-sensitiven Hydrogele eignen sich damit als chemomechanische Wandler zur Detektion verschiedener Spezies in einer Messlösung [1].

Gekoppelt mit einem mechanoelektrischen Wandler, z.B. einem piezoresistiven Drucksensor, ist aus der Volumenänderung ein elektrisches Messsignal ableitbar. In die Kavität dieses Drucksensors eingebracht, kann der Quelldruck des Hydrogels zur Erzeugung eines Messsignals genutzt werden (Abb. 1) [2]. Der Träger des Drucksensors ist im Bereich der Kavität perforiert, sodass die Messlösung hindurchtreten kann und innerhalb des Hohlraums mit dem Hydrogel wechselwirkt.

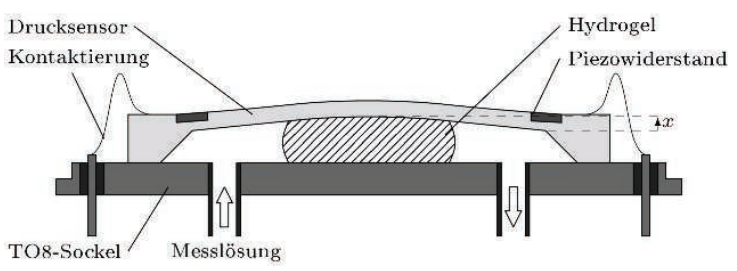

(a)

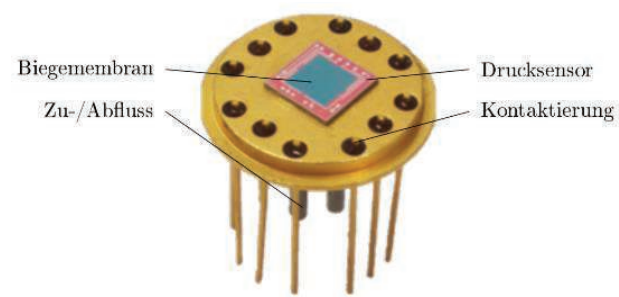

(b)

Abb. 1: Schematischer Aufbau (a) und Fotografie (b) eines Hydrogel-basierten chemischen Sensors mit einem Drucksensor als mechanoelektrischem Wandler (C41, Epcos AG) auf einem TO8-Sockel. 
Kraftkompensationsmethode

Herkömmliche Hydrogel-basierte Chemosensoren, die einen Drucksensor als mechanoelektrischen Wandler nutzen, erzeugen ein Signal nach dem Ausschlagverfahren. Nach einer Änderung des Stimulus diffundiert Lösungsmittel in das Polymernetzwerk ein oder aus dem Netzwerk in die umgebende Messlösung aus. Die damit einhergehende Volumenänderung verändert die Auslenkung der Biegemembran des Drucksensors. Im Drucksensor implantierte Piezowiderstände, die zu einer Wheatstone'schen Messbrücke verschaltet sind, wandeln die mechanische Deformation in eine Änderung der Brückendiagonalspannung um. Durch die langen Diffusionswege des Lösungsmittels treten jedoch für diesen Messaufbau lange Ansprechzeiten auf.

Die Kraftkompensation [3,4,5] stellt eine alternative Messmethode dar, mit der die Diffusionsprozesse des Lösungsmittels weitestgehend unterdrückt werden, indem eine entsprechende Kraft auf der Gegenseite der Biegemembran einer quellbedingten Auslenkung entgegenwirkt. Auf diese Weise wird das Eindiffundieren von Lösungsmittel in das Polymernetzwerk unterdrückt. In [6] wurde dazu eine Druckluftquelle genutzt, während in [7] ein temperatursensitives Hydrogel als Aktor dient. Beide Aufbauten sind jedoch komplex und schwer miniaturisierbar.

In dieser Arbeit wird daher ein neuer Ansatz der Kraftkompensation in Hydrogel-basierten Sensoren verwendet. Ist in vorhergehenden Ansätzen die temperaturgesteuerte Aktorfunktion zum Erzeugen einer Gegenkraft räumlich von der chemisch sensitiven Hydrogelschicht entkoppelt, wird hier ein bisensitives Hydrogel genutzt, das gleichzeitig die sensorische als auch die aktorische Funktion erfüllt. Letztere ist durch einen Temperatureintrag mit Hilfe eines elektrothermischen Wandlers (Peltierelement) aktivierbar (Abb. 2). Quillt das Hydrogel durch den zu messenden Stimulus, kann diesem durch Erwärmen oder Abkühlen des bisensitiven Hydrogels entgegengewirkt werden. Die Rückkopplung der Brückenausgangsspannung als regelungstechnische Ausgangsgröße und Maß für den Gleichgewichtsquellungszustand erfordert dabei die Einbettung des Sensors in einen geschlossenen Regelkreis.

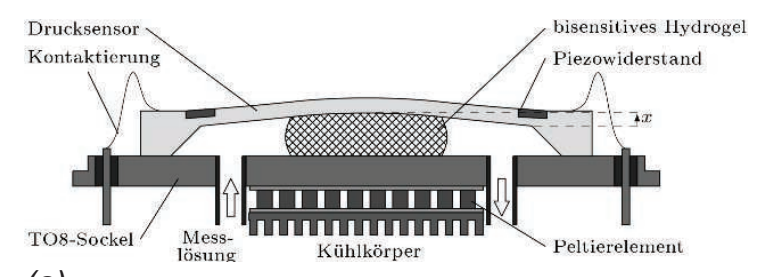

(a)

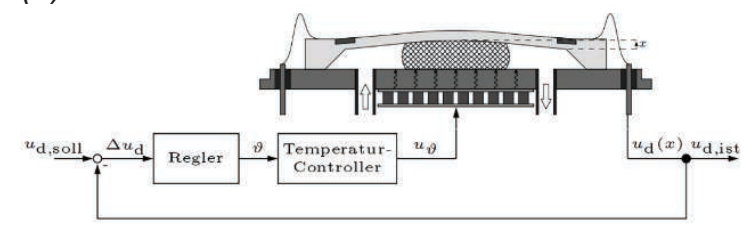

(b)

Abb. 2: Piezoresistiver chemischer Sensor mit bisensitivem Hydrogel: (a) Aufbau, (b) Einbettung des Sensors in eine Regelschleife zur Kompensation der Biegeplattenauslenkung bzw. des Hydrogelvolumens.

Die Messinformation über die Konzentration des vorliegenden Stimulus ist im Gegensatz zur Ausschlagmethode nunmehr in der Anregung der Aktorfunktion, also dem Temperatureintrag in das Hydrogel bzw. der Betriebsspannung des Peltierelements, enthalten.

Mit diesem neuartigen, intramolekularen Kompensationsverfahren vereinfacht sich der Sensoraufbau drastisch, da kein zusätzlicher Aktor auf der Gegenseite der Biegemembran notwendig ist.

$\mathrm{Da}$ das Hydrogelvolumen nahezu konstant bleibt, entfallen Diffusionsprozesse des Lösungsmittels im Hydrogel. Dies verkürzt die Ansprechzeit und verlängert die Langzeitstabilität.

\section{Bisensitives Hydrogel}

\section{Anforderungen}

Um eine intramolekulare Kraftkompensation zu ermöglichen, ergeben sich eine Reihe von Anforderungen an das bisensitive Hydrogel als chemomechanischen Wandler. Zum einen ist ein lineares Übertragungsverhalten zwischen anregender Stimulusgröße und Quellgrad wünschenswert. Zum anderen muss die Ansprechzeit des Hydrogels auf den Aktorstimulus geringer sein als auf den Sensorstimulus, um einem Überschwingen im Regelprozess entgegenzuwirken.

Diese Anforderungen erfüllt das Copolymer net-P(NiPAAm-co-AA), ein speziell für die Messung der lonenkonzentration $\mathrm{CNaCl}$ in Kochsalzlösungen und für die Anwendung im kraftkompensierten Sensor entwickeltes Hydrogel [8]. Die Sensitivität gegenüber der lonenkonzentration stellt die sensorische Komponente im Polymer dar und ist auf das Comonomer Acrylsäure AA zurückzuführen. 
Zum anderen ist der Quellgrad abhängig von der Temperatur $\vartheta$, hervorgerufen durch das Comonomer $\mathrm{N}$-Isopropylacrylamid NiPAAm, das eine untere kritische Lösungstemperatur (LCST) von $32{ }^{\circ} \mathrm{C}$ [9] besitzt. Diese Sensitivität dient der Kompensation der salzkonzentrationsbedingten Quellung und stellt damit die aktorische Funktion innerhalb der Hydrogelschicht dar. Die anionischen Netzwerkbestandteile wechselwirken mit NiPAAm derart, dass das für Poly-NiPAAm typische sprunghafte Ändern des Quellungsgrads um die untere kritische Lösungstemperatur bei $32^{\circ} \mathrm{C}$ gestreckt und so eine Linearisierung erreicht wird. Dies geschieht dadurch, dass anionische Seitengruppen lokal die LCST von NiPAAm verschieben [10]. Über die Zusammensetzung des Copolymers kann so das Quellverhalten des Makromoleküls gezielt beeinflusst werden.

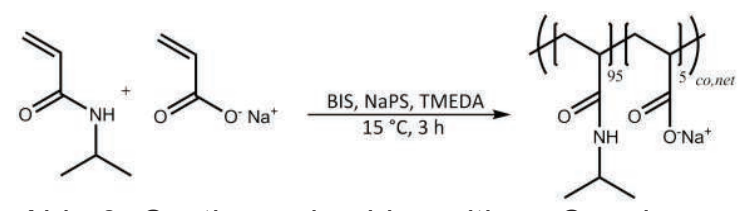

Abb. 3: Synthese des bisensitiven Copolymers net-P(NiPAAm-Co-AA) mit $95 \%$ NiPAAm und 5\% Acrylsäure. Als Vernetzer diente N,N'-Methylen-Bisacrylamid (BIS), als Initiator Natriumpersulfat (NaPS), als Katalysator $N, N, N$ ', N'-Tetramethyl-ethylendiamin (TMEDA) [8].

In [8] wurde das bisensitive Hydrogel net-P(NiPAAm-co-AA) als ein Polymer mit statistischer Verteilung mit 95\% NiPAAm und $5 \%$ AA vorgeschlagen. Abb. 3 zeigt die zugehörige Synthese und Abb. 4 das statische Quellverhalten dieses Hydrogels.

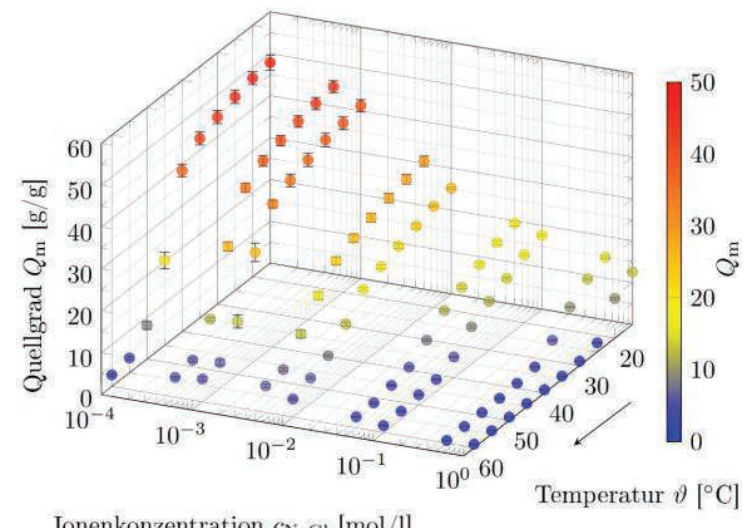

Ionenkonzentration $c_{\mathrm{NaCl}}[\mathrm{mol} / \mathrm{l}]$

Abb. 4: Statisches Quellverhalten (massenbezogener Quellgrad $Q_{m}$ ) des bisensitiven Copolymers net-P(NiPAAm-co-AA) in Abhängigkeit von der lonenkonzentration $\mathrm{CNaCl}_{\mathrm{Na}}$ einer Kochsalzlösung sowie der Temperatur $\vartheta[8]$.
Am statischen Quellverhalten ist erkennbar, dass Wertepaare von Salzkonzentration und Temperatur existieren, die den gleichen Quellgrad aufweisen. Damit ist die Kompensation einer Volumenänderung infolge sich ändernder lonenkonzentrationen über einen entsprechenden Temperatureintrag möglich.

\section{Experimentelle Untersuchungen}

Abb. 5 zeigt im Vergleich das freie Quellen des bisensitiven Hydrogels bei konstanter Temperatur $\left(50^{\circ} \mathrm{C}\right)$ und bei der jeweils zur Volumenkonstanz in Abb. 4 entsprechenden Temperatur.

In einer ersten Messung erfolgt eine sprunghafte Änderung der Salzkonzentration von $0,0001 \mathrm{~mol} / \mathrm{l}$ auf $0,05 \mathrm{~mol} / \mathrm{l}$, während die Temperatur konstant bei $50^{\circ} \mathrm{C}$ gehalten wird. Das bisensitive Hydrogel aus [8] wurde dazu in einem Glasröhrchen (Durchmesser $3 \mathrm{~mm}$ ) synthetisiert, dreimal für je $24 \mathrm{~h}$ mit destilliertem Wasser gespült und anschließend in flache Zylinder (Höhe $1 \mathrm{~mm}$ ) vereinzelt und getrocknet. Nach Trocknung wurden die flachen Hydrogelscheiben in Probengläschen mit $0,0001 \mathrm{~mol} / \mathrm{K}$ Kochsalzlösung bei $50{ }^{\circ} \mathrm{C}$ in einem Ofen (BD56, Binder $\mathrm{GmbH}$ ) für $24 \mathrm{~h}$ vorkonditioniert. Zur Sprunganregung wurden die Hydrogelproben in eine auf $50^{\circ} \mathrm{C}$ erwärmte Salzlösung von $0,05 \mathrm{~mol} / \mathrm{l}$ in einer regelbaren Temperiervorrichtung (Belektronig $\mathrm{GmbH}$ ) eingelegt. Die Aufzeichung der Sprungantwort der Hydrogelproben auf den Stimuluswechsel erfolgte mittels eines Mikroskops (Bresser $\mathrm{GmbH}$ ) und einer Kamera (MikroCam Pro, Bresser $\mathrm{GmbH}$ ). Der Quellgrad $Q_{d}$ wurde durch die relative Änderung des Durchmessers der Hydrogelscheiben beschrieben (1):

$Q_{d}=\frac{d-d_{0}}{d_{0}}$

Die Messung des Anfangsdurchmessers $d_{0}$ und des zeitabhängigen Durchmessers $d$ erfolgte mit der Software MikroCamLabll (Bresser $\mathrm{GmbH}$ )

Die zweite Messung (blau) zeigt einen gleichzeitigen Wechsel beider Stimuli. Die beiden Wertepaare sind dabei so gewählt, dass sich anhand des statischen Quellverhaltens ein nahezu identisches Volumen ergeben sollte. Als Wertepaare wurden $\left(0,0001 \mathrm{~mol} / \mathrm{l}, 50^{\circ} \mathrm{C}\right)$ und $\left(0,05 \mathrm{~mol} / \mathrm{l}, 30^{\circ} \mathrm{C}\right)$ gewählt. Für ersteres ist gemäß Abb. 4 ein massenbezogener Gleichgewichtsquellungsgrad von 14,72 $\pm 0,89$ bekannt, für zweiteres ein Quellgrad von $14,63 \pm 0,43$. Der Messaufbau entsprach dem der ersten Messung. 


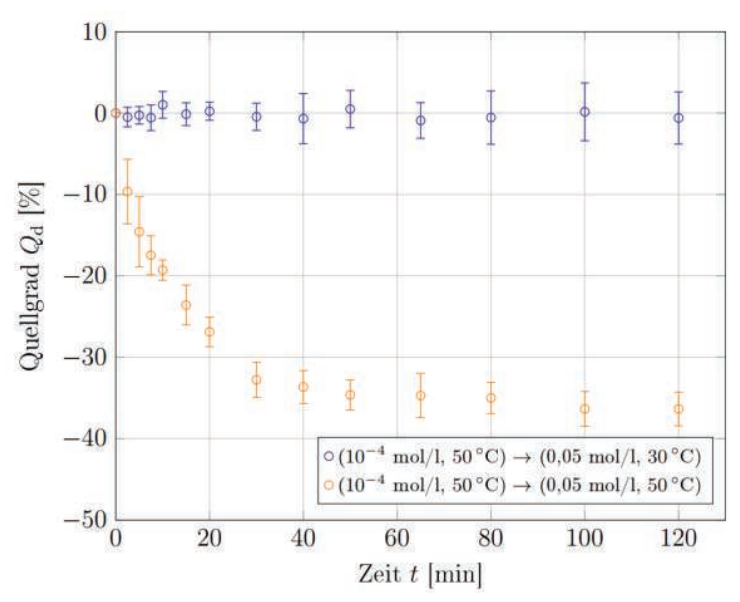

Abb. 5: Sprungantworten von Hydrogelproben des kraftkompensatorisch wirksamen Hydrogels net-P(NiPAAm-Co-AA) auf eine sprungartige Änderung der Salzkonzentration (orange) sowie auf eine das Volumen kompensierende Sprunganregung durch entgegengesetzte Stimulibeaufschlagung (blau) bei $t=0$. Die Unsicherheit der geregelten Temperatur beträgt $0,1^{\circ} \mathrm{C}$, die relative Unsicherheit der Ionenkonzentration $0,05 \%$.

Anhand der Quellverläufe ist zu erkennen, dass sich bei Änderungen der Salzkonzentration durch entsprechenden Temperatureintrag das Volumen der Hydrogelproben nahezu konstant halten kann. Die Ansprechzeit auf den Aktorstimulus ist dabei ausreichend kurz, sodass kein bzw. nur ein geringes Überschwingen eintritt. Dieses Verhalten wird durch Materialwerte für Diffusionskoeffizienten aus der Literatur bestätigt. Im Vergleich zum kooperativen Diffusionskoeffizienten der Volumenänderung von ca. $10^{-7} \mathrm{~cm}^{2} / \mathrm{s}$ sind die Diffusionskoeffizienten der beiden Stimuli mit ca. $10^{-3} \mathrm{~cm}^{2} / \mathrm{s}$ (Temperaturleitfähigkeit im Hydrogel) und $10^{-5} \mathrm{~cm}^{2} / \mathrm{s}$ (Diffusion gelöster Bestandteile in einer Messlösung, z. B. Ionen) um mindestens zwei Ordnungen größer [11,12].

Die Dynamik des intramolekular kraftkompensierten, chemischen Sensors auf Hydrogelbasis ist damit im Wesentlichen durch den Regler bestimmt, der den Temperatureintrag in das Hydrogel vorgibt.

Modelle der intramolekularen Volumenkompensation

Die Fähigkeit des bisensitiven Hydrogels zur intramolekularen Kompensation lässt sich anhand zweier Modelle beschreiben:

(a) Modell der separaten Phasen

Die Monomerblöcke im Hydrogel bilden separate Phasen, die abhängig vom Stimulus spezifisch Lösungsmittel immobilisieren. Diese Blöcke können damit als fein verteilte Lösungs- mittelspeicher betrachtet werden. Bei einem gleichzeitigen und entgegengesetzten Wechsel beider Stimuli, der wieder zum gleichen Volumenquellungsgrad führt, tauschen benachbarte Phasen Lösungsmittel aus. Die mittlere Diffusionsweglänge wird dadurch drastisch reduziert, da das Lösungsmittel im Vergleich zu einem vollständigen Quellen bzw. Entquellen bei nur einem Stimuluswechsel wesentlich kürzere Distanzen zurücklegt. Dieser Mechanismus würde das Verhalten bei sich einander durchdringenden bzw. interpenetrierenden Netzwerken aus zwei verschieden sensitiven Einzelnetzwerken oder aber bei Pfropfpolymeren, bei denen lineare Polymerketten anderer Sensitivität einem bestehenden Polymer aufgepfropft werden, erklären (Abb. 6).
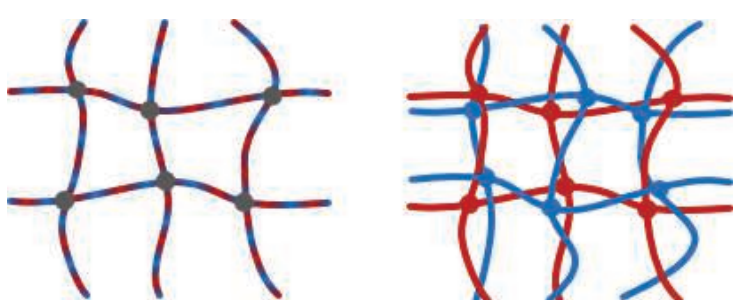

Abb. 6: Schematische Netzwerkstrukturen eines Copolymers (links) und eines interpenetrierenden Netzwerks (IPN) (rechts).

(b) Wechse/wirkungen benachbarter Comonomere

Da bei einem statistischen Copolymer, wie dem bisensitiven Hydrogel net-P(NiPAAm-Co-AA) mit einem Stoffmengenverhältnis von 95:5, die Comonomere sehr fein verteilt sind, ist ein Verhalten entsprechend dem Modell separater Phasen unwahrscheinlich. Allerdings wechselwirken die benachbarten Comonomere derart miteinander, dass die lokale Netzwerkkonfiguration keine Änderung des Quellungszustands bzw. der Lösungsmittelverteilung im Netzwerk bewirkt. Es würde somit kein Lösungsmittel im Netzwerk bewegt werden. Eine Diffusion des Lösungsmittels tritt damit weder zwischen Hydrogelumgebung und Hydrogel noch im Hydrogel selbst auf. Dies reduziert die Ansprechzeit des Hydrogels im idealen Fall so weit, dass nur die Diffusionskoeffizienten der beiden Stimuli Ionenkonzentration bzw. Temperatur relevant sind, welche um mindestens zwei Ordnungen größer sind als die des Lösungsmittels [12].

Beide Modellansätze gelten für einen gegensinnigen Stimuluswechsel, also eine ideale Kompensation. In der Realität kann solch eine ideale Kompensation allerdings nie stattfinden, da zu Beginn des Kompensationsprozesses die zu kompensierende Auslenkung noch nicht 
bekannt bzw. mit Unsicherheiten behaftet ist [3]. Im Falle eines geregelten Kompensationsbetriebs muss daher stets geringfügig Lösungsmittel in das Netzwerk eindiffundieren oder aus dem Netzwerk ausdiffundieren, damit ein Ausgangssignal für den Regler vorliegt. Dies führt zu einem Einschwingvorgang, der je nach Auslegung des Systems und des Reglers verschieden ausgeprägt ist. Letztendlich beeinflusst auch die Zeitkonstante der Temperatureinkopplung die Systemdynamik.

\section{Zusammenfassung und Ausblick}

Der Quellgrad des bisensitiven Hydrogels net-P(NiPAAm-co-AA) hängt sowohl von der Konzentration der umgebenden Kochsalzlösung als auch von der Temperatur ab. Für jede Konzentration gibt es eindeutig eine Temperatur bei der sich das initiale Volumen nicht ändert. Diese Eigenschaft erlaubt es kraftkompensierte Sensoren zur Messung der Salzkonzentration zu schaffen, bei denen über eine Temperaturregelung die konzentrationsbedingte Volumenänderung zu Null geregelt wird.

Für die Reglersynthese sind geeignete Modelle zur Streckenbeschreibung abzuleiten, die neben der thermischen Übertragungsstrecke und der Anregung auch die intrinsischen Wechselwirkungen des bisensitiven Hydrogels berücksichtigen. Aus der Systemkenntnis heraus ist ein geeigneter Reglerentwurf möglich, der eine Reduktion der Ansprechzeit des Hydrogelbasierten Sensors erlaubt.

\section{Literaturnachweis}

[1] G. Urban, G. Gerlach, K. Arndt, M. Günther, Hydrogel Sensors and Actuators - Engineering and Technology, Springer Series on Chemical Sensors and Biosensors 6, 165-193 (2009); doi: 10.1007/978-3-540-75645-3_5

[2] G. Gerlach, K. Arndt, J. Sorber, A. Richter, Sensor zur Messung der Anwesenheit und Konzentration von Komponenten in einer Flüssigkeit, DE10129987 C2 (2003)

[3] L. Krause, Vergleich von Kompensations- und Ausschlagprinzip am Beispiel der Wägetechnik, Technisches Messen 71, 643-650 (2004); doi: 10.1524/teme.71.12.643.54697

[4] L. Krause, Dynamische Wägetechnik, Wissenschaftlicher Verlag Berlin, (2005);

[5] D. Stuart-Watson, J. Tapson, Simple force balance accelerometer/seismometer based on a tuning fork displacement sensor, Review of Scientific Instruments 75, 3045-3049 (2005); doi: $10.1063 / 1.1786333$

[6] V. Schulz, Advanced Hydrogel-based Chemical Microsensors, TUDpress, (2013)

[7] K. Deng, G. Gerlach, Design, assembly and testing of a force-compensated hydrogel-based pH sensor," IMCS 2016 - The 16th International Meeting on Chemical Sensors, (2016)
[8] S. Binder, A. T. Krause, B. Voit, G. Gerlach, Bisensitive Hydrogel with Volume Compensation Properties for Force Compensation Sensors, IEEE Sensors Letters (2017), under review

[9] H. G. Schild, Poly(N-isopropylacrylamide): experiment, theory and application, Progr. Polym. Sci. 17, 163 (1992); doi: 10.1016/0079-6700(92)90023-R

[10] G. Chen, A. S. Hoffman, Macromol. Chem. Phys. 196, 1251 (1995)

[11] S. H. Gehrke, Synthesis, Equilibrium Swelling, Kinetics, Permeability and Applications of Environmentally Responsive Gels, Advances in Polymer Science 110, 81-144 (1993); doi: 10.1007/BFb0021130

[12] A. T. Krause, S. Zschoche, M. Rohn, C. Hempel, A. Richter, D. Appelhans, B. Voit, Swelling behavior of bisensitive interpenetrating polymer networks for microfluidic applications, Soft Matter 12, 5529 (2016); doi: $10.1039 / \mathrm{c} 6 \mathrm{sm} 00720 \mathrm{a}$

\section{Danksagung}

Die Autoren danken der Deutschen Forschungsgemeinschaft für die Finanzierung im Rahmen des GRK 1865 „Hydrogel-basierte Mikrosysteme“. 